\title{
Design of sliding mode observer for a class of uncertain neutral stochastic systems
}

\author{
Zhen $\mathrm{Liu}^{\mathrm{a}}{ }^{\mathrm{b}}$, Lin Zhao ${ }^{\mathrm{c}}$, Quanmin Zhu ${ }^{\mathrm{d} *}$ and Cunchen $\mathrm{Gao}^{\mathrm{a}}$ \\ ${ }^{a}$ School of Mathematical Sciences, Ocean University of China, Qingdao, China; ${ }^{b}$ Institute for Sustainable \\ Manufacturing, College of Engineering, University of Kentucky, Lexington, USA; ${ }^{c}$ College of Automation \\ Engineering, Qingdao University, Qingdao, China; ${ }^{d}$ Department of Engineering Design and Mathematics, \\ University of the West of England, Bristol, $U K$
}

(Received 00 Month 20XX; final version received 00 Month 20XX)

\begin{abstract}
The problem of robust $\mathscr{H}_{\infty}$ control for a class of uncertain neutral stochastic systems (NSS) is investigated by utilizing the sliding mode observer (SMO) technique. This paper presents a novel observer and integral-type sliding surface design, based on which a new sufficient condition guaranteeing the resultant sliding mode dynamics (SMDs) to be mean-square exponentially stable with a prescribed level of $\mathscr{H}_{\infty}$ performance is derived. Then, an adaptive reaching motion controller is synthesized to lead the system to the predesigned sliding surface in finite-time almost surely. Finally, two illustrative examples are exhibited to verify the validity and superiority of the developed scheme.
\end{abstract}

Keywords: neutral stochastic systems; sliding mode control; non-fragile observer; $\mathscr{H}_{\infty}$ performance; stability

\section{Introduction}

Neutral systems, where the delays exist in both the system state and the state derivative, have been received great attention in the control community during the past decades, and arise in various applications (Hale, \& Verduyn Lunel, 1993), e.g., the lossless transmission system, simultaneously. Thus, a great amount of studies have been devoted to the systems from a practical point of view, for instance, stability analysis (Karimi, 2011; Sakthivel, Mathiyalagan, \& Anthoni, 2012) and control designs, which include $\mathscr{H}_{\infty}$ filtering (Shen, Xu, Zhou, \& Lu, 2011), guaranteed cost control (Parlakc, 2010), observer design (Elhsoumi, Ali, Bel, Harabi, \& Abdelkrim, 2016), etc. In fact, for the more general case where the control matrix $B$ is perturbed by some factors, i.e., $B(x, \chi, t)$, where $\chi$ is an uncertain parameter vector (Hung, Gao, \& Hung, 1993), the following uncertain neutral system is taken as example:

$$
\dot{x}(t)-D \dot{x}(t-\tau)=A(t) x(t)+A_{d}(t) x(t-d)+B(x, \chi, t) u(t)+G v(t)
$$

where $B(x, \chi, t) \triangleq B+\Delta B(\chi, t)+g(x, \chi, t)$ with the form of $\Delta B(\chi, t)$ and $g(x, \chi, t)$ as

$$
\Delta B(\chi, t)=B \Delta \tilde{B}(\chi, t), g(x, \chi, t)=B \Delta \tilde{g}(x, \chi, t)
$$

for certain $\Delta \tilde{B}(\chi, t)$ and $\Delta \tilde{g}(x, \chi, t)$. Here, we recall the physical meaning of (2) is that all modeling uncertainties and disturbances enter the system through the control channel, i.e., the matched uncertainties in sliding mode control (SMC) theory (Hung et al., 1993). Thus, in the position, when subjected to some perturbations or nonlinearities through the control channel of the system, the aforementioned control design methods may lose their reliable effects upon many occasions. 
Conversely, SMC (Ahmad, \& Zhu, 2015; Utikin, 1992), because of its various attractive features such as quick response, good transient performance, particularly, the valuable invariance against matched uncertainties, has been well known as an effective robust control strategy and also has found to be widely applied to various complex systems (Basin, Ferreira, \& Fridman, 2007; Liu, \& Gao, 2016; Wu, \& Lam, 2008; Zhao, $\&$ Zhu, 2014) as well in many technical problems (Zhao, \& Jia, 2015). As for the neutral systems, some excellent works have been reported. To name a few, SMC for uncertain neutral systems with structural uncertainties has been studied in Niu, Lam, \& Wang (2004). In Gao, Liu, \& Xu (2013), the problem of robust exponential stability for a class of uncertain neutral systems has been considered on the basis of an integral SMC approach. It is observed that, in spite of the availability of SMC, however, most of the reports are obtained upon the premise that all the system states are accessible. In many practical systems, it has been approved that the system states may not be totally acquired and even generally not easily to be evaluated through output measurement. In order to tackle these problems, by incorporating the superiority of the SMC with the observer effectively, the observer-based SMC technique, also called as the sliding mode observer (SMO) strategy (Rahme, \& Meskin, 2015; Shi, Liu, \& Zhang, 2015), has been developed to solve the state estimation issue for a variety of complex systems as well many realistic engineering plants successfully, see Kao, Li, \& Wang (2014); Li, Shi, Yao, \& Wu (2016); Liu, Gao, \& Kao (2015); Wu, Wang, \& Zeng (2008); Yan, Spurgeon, \& Edwards (2010); Zhang, Shi, \& Lin (2016) and the references therein. For instance, the SMO design for nonlinear uncertain neutral systems with unmeasured states has been perfectly investigated in $\mathrm{Wu}$ et al. (2008); afterwards, the observer-based $\mathscr{H}_{\infty}$ control scheme has been proposed for uncertain neutral systems via integral SMC in Liu et al. (2015).

On another research front, stochastic perturbations often exist in many real-world systems, and the additive stochastic effects result in a type of neutral stochastic systems (NSS) (Mao, 2007), see Eq. (3) in this paper, which play an important role in many industrial fields. In recent years, increasing efforts have been made to probe the NSS, the main research of which focuses on the stability analysis (e.g., stochastic stability and stability in the mean-square sense, etc) and control of the systems, e.g., Chen, Hu, \& Wang (2014); Chen, \& Shen (2009); Chen, Zheng, \& Shen (2009); Chen, Zheng, \& Xue (2010); Huang, \& Mao (2009); Jankovic, Randjelovic, \& Jovanovic (2009); Song, Xu, Xia, Zou, \& Chen (2011); Song, Park, Wu, \& Zhang (2013); Xu, Chu, Lu, \& Zou (2006); Xu, Shi, Chu, \& Zou (2006) and the references therein. It is noted that, SMC for uncertain NSS without matched uncertainties have been concerned by Chen, \& Zhang (2008); Kao, Wang, Xie, Karimi, \& Li (2015). Nevertheless, when uncertainties and/or perturbations appear through the control channels, less research work has been involved on the NSS, which forms the first motivation of our concern.

In addition, consider that the system states may not be accessible on account of the factors (e.g., cost, technique, etc), so the SMO scheme shall be employed to deal with this case. More specifically, the observer-based SMC problem has been investigated for a class of NSS without the matched uncertainties, see Kao, Xie, Wang, \& Karimi (2015); Li, \& Li (2009), whereas the uncertainties are considered in the present work. Also, robust adaptive SMC for uncertain neutral Markovian jumped systems with unmeasured states and unknown nonlinearity has been studied in Yao, Liu, Li, \& Ma (2015), however, the state-dependent stochastic effect is not considered due to some difficulties therein. To the best of the authors knowledge, the issue remains open and challenging, and some problems may be still questions of common interest, then we hope to shorten such a gap, which is the second motivation of the paper. It should also be mentioned that, the matched ones has been done for deterministic neutral systems, where the general method is that: Both the controller itself and a discontinuous output error injection term (or called the controller compensator) are required to design for rejecting the uncertainties and guaranteeing robust stability of the closed-loop systems. In such case, the associated problems including the sliding surface design will be more complicated to configure. Moreover, because the techniques for deterministic neutral systems such as Liu et al. (2015); Wu et al. (2008) are not directly applicable to the NSS, another way should be adopted.

To respond the above situations, the $\mathscr{H}_{\infty}$ performance and mean-square exponential stability problem for a class of NSS with structural uncertainties, perturbation and external disturbance is investigated via a novel SMO scheme in this paper. The main contribution of the work is outlined below:

1). New technical route of the developed scheme that is to be clearly presented. 
In detail, for most of the existing methods to handle the observer-based issues, main ideas are that: System stability is analyzed through the original system and its observer so as to ensure the error system to converge to the equilibrium state. Here, the new technical route is that: If the stability of the original system and its error system can be guaranteed, the observer states can tend to be stable as it is. Namely, the control problem will be achieved through the original system and its error system in the present scheme.

2). A particular nonfragile observer is established as the new SMO.

It is noted that, the SMO design, no matter for the deterministic neutral systems or the existing NSS, is generally developed with the so-called composite controller, i.e., the controller itself and its compensator (or the discontinuous output error injection term) are both required to satisfy the main goal. However, in this note, the composite controller will be no longer needed for SMO, and the resultant convenience is that: The controller is only performed in the original system part, and the actions of the error system and the observer will be responded automatically.

3). A novel single sliding surface is introduced for the scheme.

Compared with the previous designs, error terms are introduced in the present sliding surface design, which well performs to avoid difficulties caused by the perturbations through the control channel. This design may also benefit to highlight the attractive feature of SMC that the SMDs could be insensitive to all matched uncertainties. The design of the sliding surface for the NSS may be more interesting via the layout.

4). A novel adaptive sliding mode controller is presented.

Due to the state-dependent stochastic noises in the NSS, the techniques for deterministic neutral systems may not be directly applicable to the NSS. At this point, an adaptive controller is synthesized, where the outputs of the systems and its observer are involved, and the unknown bounds can be exactly tracked. Then, the expected performance of the closed-loop systems can be achieved.

The paper is organized as follows. Problem description and preliminaries are given in Section 2. Section 3 is arranged by the main results. Illustrative examples are provided to verify the theoretical result in Section 4. Conclusions are epitomized in Section 5.

Notation. Throughout the paper, the notation $X>Y$ (respectively, $X \geq Y$ ) means that the matrix $X-Y$ is positive definite (respectively, positive semi-definite). $\left(\Omega, \mathscr{F},\left\{\mathscr{F}_{t}\right\}_{t \geq 0}, \mathscr{P}\right)$ represents a completed probability space with a natural filtration $\left\{\mathscr{F}_{t}\right\}_{t \geq 0}$, where $\Omega$ is a sample space, $\mathscr{F}$ is the $\sigma$-algebra of subset of the sample space, and $\mathscr{P}$ is the probability measure on $\mathscr{F}$. Let $h>0$ and $\mathscr{C}\left([-h, 0] ; \mathbb{R}^{n}\right)$ denote the family of all continuous $\mathbb{R}^{n}$-valued functions on $[-h, 0]$. Let $\mathscr{C}_{\mathscr{F}_{0}}^{b}\left([-h, 0] ; \mathbb{R}^{n}\right)$ be the family of all $\mathscr{F}_{0}$-measurable bounded $\mathscr{C}\left([-h, 0] ; \mathbb{R}^{n}\right)$-valued random variables. $\mathscr{E}\{\cdot\}$ is the expectation operator with respect to some probability measure $\mathscr{P}$. The superscript "T" denotes the transpose of a vector or matrix, and the symmetric elements of the matrix is denoted by “*”. $\operatorname{sym}\{X\}$ is denoted as $\operatorname{sym}\{X\}=X+X^{\mathrm{T}}$. If $M$ is a matrix, its operator norm is denoted by $\|M\|=\sup \{\|M x\|:\|x\|=1\}, \lambda_{\max }(M)$ and $\lambda_{\min }(M)$ represent its maximum and minimum eigenvalues, respectively. $\operatorname{Tr}\{\cdot\}$ denotes the trace of a matrix. $\operatorname{diag}\{\cdot\}$ represents a block-diagonal matrix. $\mathscr{L}_{2}[0, \infty)$ stands for the space of square integral vector functions over $[0, \infty)$.

\section{System description and preliminaries}

Consider the following $n$-dimensional neutral stochastic systems (NSS) described by

$$
\left\{\begin{aligned}
\mathrm{d}[x(t)-D x(t-\tau)]=\left\{(A+\Delta A(t)) x(t)+\left(A_{d}+\Delta A_{d}(t)\right) x(t-d(t))\right. & \\
& +B[u(t)+f(t, x(t))]+G v(t)\} \mathrm{d} t+g(t, x(t)) \mathrm{d} \omega(t) \\
y(t)= & C x(t) \\
x(\theta)= & \phi(\theta), \theta \in[-h, 0]
\end{aligned}\right.
$$

where $x(t) \in \mathbb{R}^{n}$ is the state vector, $u(t) \in \mathbb{R}^{m}$ is the control input, $y(t) \in \mathbb{R}^{q}$ is the system output. $\tau>0$ is a constant neutral-term time-delay, $d(t)$ is the time-varying delay, which satisfies $0<d(t) \leq d$ and $\dot{d}(t) \leq \mu<1$, where $d$ and $\mu$ are constants, and $h=\max \{\tau, d\} . \omega(t)$ is a standard scalar Brownian motion 
defined on a completed probability space $\left(\Omega, \mathscr{F},\left\{\mathscr{F}_{t}\right\}_{t \geq 0}, \mathscr{P}\right)$ with a natural filtration $\left\{\mathscr{F}_{t}\right\}_{t \geq 0}$, and satisfies $\mathscr{E}\{\mathrm{d} \omega(t)\}=0, \mathscr{E}\left\{\mathrm{d} \omega^{2}(t)\right\}=\mathrm{d} t . v(t) \in \mathrm{R}^{l}$ represents a set of exogenous disturbance which belongs to $\mathscr{L}_{2}[0, \infty) . \phi(t) \in \mathscr{C}_{\mathscr{F}_{0}}^{b}\left([-h, 0] ; \mathbb{R}^{n}\right)$ is the initial condition. $A, A_{d}, B, C, D$ and $G$ are known real matrices, $B$ is of full column rank, and the spectrum radius of the matrix $D$, i.e., $\rho(D)$, satisfies $\rho(D)<1$. To facilitate the result, the following preliminaries are introduced for the system (3).

Assumption 1. The structural uncertainties $\Delta A(t)$ and $\Delta A_{d}(t)$ are norm bounded, i.e., $\left[\Delta A(t) \Delta A_{d}(t)\right]=$ $E J(t)\left[\begin{array}{ll}F & F_{d}\end{array}\right]$, where $E, F$ and $F_{d}$ are constant matrices, and $J(t)$ is unknown matrix function satisfying $J^{\mathrm{T}}(t) J(t) \leq I$ for all $t \geq 0$.

Assumption 2. $f(t, x)$ is unknown nonlinearity which represents the lumped perturbation of a physical plant through the control channel satisfying $\|f(t, x)\| \leq \alpha\|y(t)\|$, where $\alpha>0$ is an unknown constant (Li et al., 2016; Yao et al., 2015).

Assumption 3. The diffusion gain function $g(t, x)$ may not be exactly known but there exist a matrix $M$ such that the inequality holds: $\operatorname{Tr}\left\{g^{\mathrm{T}}(t, x) g(t, x)\right\} \leq\|M y(t)\|^{2}$.

Remark 1: The Assumption 3 is reasonable to a certain degree. Actually, the state-dependent stochastic noises $g(t, x)$ may not be accessible but could be evaluated by $\operatorname{Tr}\left\{g^{\mathrm{T}}(t, x) g(t, x)\right\} \leq\|N x(t)\|^{2}$, where $N$ is a constant matrix, see Huang, \& Mao (2010); Kao et al. $(2014,2015)$. With the relevance that $y(t)=C x(t)$, i.e., $x(t)=C^{+} y(t)$, the assumption is easily introduced herein, where $C^{+}$denotes the Moore-Penrose inverse of $C$. Consider that $x(t)$ may be unmeasured, the output information is used to facilitate the control design. As to $C$, its selection may be flexible by the actual design, e.g., it can be of full column rank.

Based on above conditions, one can verify that the stochastic neutral system (3) with $u(t)=0$ has a unique solution according to Huang et al. (2009); Mao (2007). In fact, denote the following terms:

$$
m(t)=(A+\Delta A(t)) x(t)+\left(A_{d}+\Delta A_{d}(t)\right) x(t-d(t))+B f(t, x)+G v(t), \quad n(t)=g(t, x)
$$

for all $t \geq 0$. It is easy to observe that $\|m(t)\|^{2} \leq K_{m}\left\|x_{t}\right\|^{2},\|n(t)\|^{2} \leq K_{n}\left\|x_{t}\right\|^{2}$, where $x_{t}=\{x(t+\theta):-h \leq$ $\theta \leq 0\}, K_{m}$ and $K_{n}$ are positive and can be found upon the premise of each component of $m(t)$ and $n(t)$, respectively. Thus, this implies $m(t)$ and $n(t)$ satisfy the local Lipschitz condition and the linear growth condition. At this point, there exists a unique continuous solution expressed by $x(t ; \phi)$ to the NSS (3), and the details can refer to Theorem 3.1 of Mao (2007).

Definition 1: Chen et al. (2010): The system (3) is said to be mean-square exponentially stable if there exist scalars $\eta>0, \beta>0$ such that $\mathscr{E}\left\{\|x(t)\|^{2}\right\} \leq \eta e^{-\beta t} \sup _{-h \leq \theta \leq 0} \mathscr{E}\left\{\|\phi(\theta)\|^{2}\right\}$ for all admissible uncertainties.

Lemma 2.1: Huang et al. (2010): For a pair of constant matrices $G \in \mathbb{R}^{p \times p}$ and $M \in \mathbb{R}^{p \times q}$, if $G \geq 0$, then $\operatorname{Tr}\left(M^{\mathrm{T}} G M\right) \leq \lambda_{\max }(G) \operatorname{Tr}\left(M^{\mathrm{T}} M\right)$.

\section{Design of the sliding mode observer}

In this section, we will specialise in the stability analysis and controller synthesis of the closed-loop systems based on a new state observer design. The research framework and basic contents are as follows, respectively. 


\subsection{Non-fragile state observer and novel sliding surface design}

Firstly, the state observer technique is utilized to generate the accurate state estimation of the system (3). Here, the following non-fragile observer is employed for the design

$$
\left\{\begin{array}{l}
\mathrm{d}[\hat{x}(t)-D \hat{x}(t-\tau)]=\left\{A \hat{x}(t)+A_{d} \hat{x}(t-d(t))+(L+\Delta L(t))(y(t)-C \hat{x}(t))\right\} \mathrm{d} t \\
\hat{y}(t)=C \hat{x}(t) \\
\hat{x}(\theta)=\hat{\phi}(\theta), \theta \in[-h, 0]
\end{array}\right.
$$

where $\hat{x}(t) \in \mathbb{R}^{n}$ represents the estimation of $x(t), \hat{y}(t)$ denote the output of the observer. $L \in \mathbb{R}^{n \times p}$ is the observer gain to be designed later, and $\Delta L(t)$ is an additive gain variation satisfying $\|\Delta L(t)\| \leq \delta$, where $\delta>0$ is a constant, namely, the observer may be affected by some perturbations (Kao et al., 2014, 2015).

Let the estimation error be $e(t)=x(t)-\hat{x}(t)$. Thus, by subtracting (4) from (3), one can get the following estimation error system

$$
\left\{\begin{array}{c}
\mathrm{d}[e(t)-D e(t-\tau)]=\left\{[A-L C-\Delta L(t) C] e(t)+A_{d} e(t-d(t))+\Delta A(t) x(t)\right. \\
\left.\quad+\Delta A_{d}(t) x(t-d(t))+B[u(t)+f(t, x(t))]+G v(t)\right\} \mathrm{d} t+g(t, x(t)) \mathrm{d} \omega(t), \\
y_{e}(t)=C e(t)
\end{array}\right.
$$

where $y_{e}(t)$ denotes the output of the error system.

Remark 2: In this work, a particular non-fragile observer is proposed for the system. Different from previous designs of the observer, see Elhsoumi et al. (2016); Kao et al. (2014, 2015); Li et al. (2016, 2009); Lin, Wang, Lee, He, \& Chen (2008); Liu et al. (2015); Qiao, Zhang, Zhu, \& Zhang (2009); Rahme et al. (2015); Shi et al. (2015); Wu et al. (2008); Yan et al. (2010); Yao et al. (2015); Zhang et al. (2016) for details, it is worth noting that the control input and/or its compensator are both required to involve into their observer design, however, only an observer gain $L$ is to be determined here. Thus, this simplified the procedure of the state observer design, which is the first advantage of this paper.

Now, a novel integral-type sliding surface function is defined as follows:

$$
s(t)=H[e(t)-D e(t-\tau)]+H[\hat{x}(t)-D \hat{x}(t-\tau)]-\int_{0}^{t} H(A+B K) \hat{x}(\theta) \mathrm{d} \theta,
$$

where the gain matrix $K$ is to be determined. $H \in \mathbb{R}^{m \times n}$ is a known matrix satisfying $H B$ is non-singular. And it is assumed that the matrix $H$ matches the following requirement:

$$
H=N_{1} C, \quad H D=N_{2} C
$$

where $N_{1}$ and $N_{2}$ are matrices to be determined. In order to enhance the freedom of the control scheme, we introduce $H=B^{\mathrm{T}} Z$ with any $Z>0$ to benefit the entire goal.

Remark 3: From the above discussion, the problem of unknown matrices $N_{1}$ and $N_{2}$ should be solved so that the sliding surface function (6) can be expressed by

$$
s(t)=N_{1}[y(t)-\hat{y}(t)]-N_{2}[y(t-\tau)-\hat{y}(t-\tau)]+H[\hat{x}(t)-D \hat{x}(t-\tau)]-\int_{0}^{t} H(A+B K) \hat{x}(\theta) \mathrm{d} \theta,
$$

if the condition (7) is exploited. In detail, it is seen that the solution of $N_{1}$ and $N_{2}$ depends on $C, D$ and $H$ which are determined by the designer. Moreover, for practical applications, a more general algorithm which determines $N_{1}$ and $N_{2}$ will be developed in Remark 8, and the feasibility of the scheme will be validated by an example. From the point of view of practice, it is observed that the sliding surface can be accessibly designed in this work. 


\subsection{Exponential stability analysis of the SMDs with $\mathscr{H}_{\infty}$ performance}

In light of the trivial solution of the original system (3) and the error system (5), it could definitely be seen that $s(t)$ is an Ito stochastic process satisfying

$$
\begin{aligned}
\mathrm{d} s(t) & =H \mathrm{~d}[e(t)-D e(t-\tau)]+H \mathrm{~d}[\hat{x}(t)-D \hat{x}(t-\tau)]-H(A+B K) \hat{x}(t) \mathrm{d} t \\
& =\mathcal{L} s(t) \mathrm{d} t+H g(t, x(t)) \mathrm{d} \omega(t)
\end{aligned}
$$

where

$$
\begin{aligned}
\mathcal{L} S(t)= & H A e(t)+H A_{d} x(t-d(t))+H \Delta A(t) x(t)+H \Delta A_{d}(t) x(t-d(t))+H B[u(t)+f(t, x)] \\
& +H G v(t)-H B K \hat{x}(t) .
\end{aligned}
$$

To achieve the sliding motion, an equivalent controller will be derived via the SMC theory (Huang et al., 2010; Utikin, 1992), i.e., $\mathscr{E} s(t)=0$ and $\frac{\mathrm{d}(\mathscr{E} s(t))}{\mathrm{d} t}=0$, that is to say, $\mathcal{L} s(t)=0$ should be ensured from the condition $\mathscr{E}\{\mathrm{d} \omega(t)\}=0$. Therefore, the so-called equivalent controller can be obtained by

$$
\begin{aligned}
u_{e q}(t)= & K \hat{x}(t)-f(t, x)-(H B)^{-1}\left[H A e(t)+H A_{d} x(t-d(t))+H \Delta A(t) x(t)\right. \\
& \left.+H \Delta A_{d}(t) x(t-d(t))+H G v(t)\right] .
\end{aligned}
$$

Substituting (9) into the system (3), one gets the dynamic equation of the original system (3) in the sliding mode as follows

$$
\begin{aligned}
\mathrm{d}[x(t)-D x(t-\tau)]= & \left\{\left[A+B K+B_{H} \Delta A(t)\right] x(t)+B_{H}\left[A_{d}+\Delta A_{d}(t)\right] x(t-d(t))\right. \\
& \left.-\left(B K+B_{A}\right) e(t)+G_{B} v(t)\right\} \mathrm{d} t+g(t, x) \mathrm{d} \omega(t)
\end{aligned}
$$

where $B_{H}=I-B(H B)^{-1} H, B_{A}=B(H B)^{-1} H A, G_{B}=B_{H} G$. Similarly, together with (9) and (5), the related dynamic equation of the error system (5) in the sliding mode can be described by

$$
\begin{aligned}
\mathrm{d}[e(t)-D e(t-\tau)]=\left\{\left[A_{B}-L C-B K-\Delta L(t) C\right] e(t)+A_{d} e(t-d(t))+\left[B K+B_{H} \Delta A(t)\right] x(t)\right. \\
\\
\left.+\left[B_{H} \Delta A_{d}(t)-B_{d}\right] x(t-d(t))+G_{B} v(t)\right\} \mathrm{d} t+g(t, x) \mathrm{d} \omega(t)
\end{aligned}
$$

where $A_{B}=A-B_{A}, B_{d}=B(H B)^{-1} H A_{d}$.

Based on the above statement, it follows that (10) and (11) can be acknowledged as the sliding mode dynamics (SMDs) of the closed-loop systems. Then, the stability of the system (3) will be investigated through the SMDs (10)-(11) by resorting to adaptive SMC.

Remark 4: In this part, a novel integral-type sliding surface function in (6) is constructed. What really makes the design special can be listed in the following two aspects:

(i) Different from the forms proposed in Chen et al. (2008); Gao et al. (2013); Kao et al. (2015); Li et al. (2009); Liu et al. (2015); Niu (2004); Yao et al. (2015), the error terms $e(t)$ and $D e(t-\tau)$ are introduced in the sliding surface design, which thoroughly performs to avoid difficulties caused by the perturbations through the control channel (i.e., $f(t, x)$ ), as can be seen from the derivative of the SMDs (10)-(11). This design may also benefit to highlight the attractive feature of SMC that SMDs can be insensitive to all matched uncertainties;

(ii) It is worth mentioning that, two sliding surfaces or auxiliary terms (e.g., $\left.s_{e}(t)\right)$ are developed in both the estimation space and the error space in Wu et al. (2008); Yao et al. (2015), respectively, while the single integral sliding surface is designed in this scheme, which depends on the state estimate and the system output in the paper. 
In conclusion, the design of the sliding surface for NSS is simplified via the present layout, which is seen as the second advantage of the paper.

Remark 5: It should be mentioned that, as the observer (4) is applied, the control input $u(t)$ is embedded into the error system (5) as virtual controller. By utilizing the SMC method, stability analysis of the original system (3) is transformed into that of the overall closed-loop systems composed of the system (3) and its error system (5) in the sliding mode at the same time, so as to achieve the aim of the entire system control. This technical route is different from that of the existing SMO scheme, please refer to Kao et al. (2014, 2015); Li et al. (2016, 2009); Liu et al. (2015); Qiao et al. (2009); Rahme et al. (2015); Shi et al. (2015); Wu et al. (2008); Yan et al. (2010); Yao et al. (2015); Zhang et al. (2016) for more details, which may be the third merit in the paper.

Specifically, the objective of robust $\mathscr{H}_{\infty}$ performance analysis studied in this note can be summarized as two purposes: on the basis of adaptive SMO method such that:

(P1) Given a positive scalar $\gamma>0$, the following $\mathscr{H}_{\infty}$ performance index is satisfied

$$
\mathscr{E}\left\{\sup _{0 \neq v(t) \in \mathscr{L}_{2}[0, \infty)}\left\|y_{e}(t)\right\|_{2} /\|v(t)\|_{2}\right\}<\gamma
$$

under zero initial conditions;

(P2) The SMDs (10)-(11) is mean-square exponentially stable with $v(t)=0$.

In this work, we call the SMDs in (10)-(11) satisfying (P1) and (P2) is mean-square exponentially stable with $\mathscr{H}_{\infty}$ disturbance attenuation level $\gamma$. In terms of LMIs, the following sufficient criteria which guarantees the desirable performance can be derived.

Theorem 3.1: Consider the sliding surface function defined in (6) and the SMDs in (10)-(11). Given a scalar $\gamma>0$, the SMDs is mean-square exponentially stable with $\mathscr{H}_{\infty}$ disturbance attenuation level $\gamma$, if there exist symmetric definite matrices $P, Q_{1}, Q_{2}, R_{1}$, and $R_{2}$, matrices $X$ and $Y$, positive scalars $\varrho$ and $\varepsilon_{i}$ $(i=1, \ldots, 6)$ satisfying the following LMIs:

$$
\left[\begin{array}{cccccccc}
\Pi_{11} & \Pi_{12} & \Pi_{13} & \Pi_{14} & -X^{\mathrm{T}} D & 0 & P G_{B} & \Upsilon_{1} \\
* & -\tau Q_{1} & \Pi_{23} & \Pi_{24} & 0 & 0 & -D^{\mathrm{T}} P G_{B} & \Upsilon_{2} \\
* & * & \Pi_{33} & -\left(P B_{d}\right)^{\mathrm{T}} & \left(P B_{d}\right)^{\mathrm{T}} D & 0 & 0 & 0 \\
* & * & * & \Pi_{44} & \Pi_{45} & P A_{d} & P G_{B} & \Upsilon_{3} \\
* & * & * & * & -\tau R_{1} & -D^{\mathrm{T}} P A_{d} & -D^{\mathrm{T}} P G_{B} & \Upsilon_{4} \\
* & * & * & * & * & \Pi_{66} & 0 & 0 \\
* & * & * & * & * & * & -\gamma^{2} I & 0 \\
* & * & * & * & * & * & * & \Upsilon_{5}
\end{array}\right]<0
$$

where $\Pi_{11}=\operatorname{sys}\{P A+X\}+\tau Q_{1}+d Q_{2}+2 \varrho C^{\mathrm{T}} M^{\mathrm{T}} M C+\left(\varepsilon_{1}+\varepsilon_{2}+\varepsilon_{3}+\varepsilon_{4}\right) F^{\mathrm{T}} F, \Pi_{12}=-(P A+X)^{\mathrm{T}} D$, $\Pi_{13}=P B_{H} A_{d}+\left(\varepsilon_{1}+\varepsilon_{2}+\varepsilon_{3}+\varepsilon_{4}\right) F^{\mathrm{T}} F_{d}, \Pi_{14}=-X-P B_{A}+X^{\mathrm{T}}, \Pi_{23}=-D^{\mathrm{T}} P B_{H} A_{d}, \Pi_{24}=D^{\mathrm{T}}\left(X+P B_{A}\right)$, $\Pi_{33}=-d(1-\mu) Q_{2}+\left(\varepsilon_{1}+\varepsilon_{2}+\varepsilon_{3}+\varepsilon_{4}\right) F_{d}^{\mathrm{T}} F_{d}, \Pi_{44}=\operatorname{sys}\left\{P A_{B}-Y C-X\right\}+\tau R_{1}+d R_{2}+C^{\mathrm{T}} C+\left(\varepsilon_{5}+\varepsilon_{6}\right) \delta^{2} C^{\mathrm{T}} C$, $\Pi_{45}=\left(-P A_{B}+Y C+X\right)^{\mathrm{T}} D, \Pi_{66}=-d(1-\mu) R_{2}, \Upsilon_{1}=\left[\begin{array}{lllllll}P B_{H} E & 0 & 0 & 0 & 0 & 0\end{array}\right], \Upsilon_{2}=\left[\begin{array}{llllll}0 & D^{\mathrm{T}} P B_{H} E & 0 & 0 & 0 & 0\end{array}\right]$, $\Upsilon_{3}=\left[\begin{array}{llllll}0 & 0 & P B_{H} E & 0 & P & 0\end{array}\right], \Upsilon_{4}=\left[\begin{array}{llllll}0 & 0 & 0 & D^{\mathrm{T}} P B_{H} E & 0 & D^{\mathrm{T}} P\end{array}\right], \Upsilon_{5}=\operatorname{diag}\left\{-\varepsilon_{1} I,-\varepsilon_{2} I,-\varepsilon_{3} I,-\varepsilon_{4} I,-\varepsilon_{5} I,-\varepsilon_{6} I\right\}$. Moreover, the gain matrices are computed by $K=B^{+} P^{-1} X$, and $L=P^{-1} Y$. 
Proof. To begin with, choose the following Lyapunov function candidate

$$
\begin{aligned}
V(t)= & {[x(t)-D x(t-\tau)]^{\mathrm{T}} P[x(t)-D x(t-\tau)]+\tau \int_{t-\tau}^{t} x^{\mathrm{T}}(\theta) Q_{1} x(\theta) \mathrm{d} \theta+d \int_{t-d(t)}^{t} x^{\mathrm{T}}(\theta) Q_{2} x(\theta) \mathrm{d} \theta } \\
& +[e(t)-D e(t-\tau)]^{\mathrm{T}} P[e(t)-D e(t-\tau)]+\tau \int_{t-\tau}^{t} e^{\mathrm{T}}(\theta) R_{1} e(\theta) \mathrm{d} \theta+d \int_{t-d(t)}^{t} e^{\mathrm{T}}(\theta) R_{2} e(\theta) \mathrm{d} \theta .
\end{aligned}
$$

In the light of Itô differential formula (Mao, 2007), the stochastic differential of $V(t)$ is obtained as

$$
\mathrm{d} V(t)=\mathcal{L} V(t) \mathrm{d} t+2[x(t)-D x(t-\tau)+e(t)-D e(t-\tau)]^{\mathrm{T}} P g(t, x(t)) \mathrm{d} \omega(t)
$$

with the infinitesimal operator

$$
\begin{aligned}
\mathcal{L} V(t)= & 2[x(t)-D x(t-\tau)]^{\mathrm{T}} P(A+B K) x(t)+B_{H} A_{d} x(t-d(t))-\left(B K+B_{A}\right) e(t) \\
& \left.+G_{B} v(t)\right]+2[x(t)-D x(t-\tau)]^{\mathrm{T}} P B_{H}\left[\Delta A(t) x(t)+\Delta A_{d}(t) x(t-d(t))\right] \\
& +\tau x^{\mathrm{T}}(t) Q_{1} x(t)-\tau x^{\mathrm{T}}(t-\tau) Q_{1} x(t-\tau)+d x^{\mathrm{T}}(t) Q_{2} x(t)-d(1-\dot{d}(t)) \\
& \cdot x^{\mathrm{T}}(t-d(t)) Q_{2} x(t-d(t))+2[e(t)-D e(t-\tau)]^{\mathrm{T}} P\left[\left(A_{B}-L C-B K\right) e(t)\right. \\
& \left.+A_{d} e(t-d(t))+B K x(t)-B_{d} x(t-d(t))+G_{B} v(t)\right]+\tau e^{\mathrm{T}}(t) R_{1} e(t) \\
& -\tau e^{\mathrm{T}}(t-\tau) R_{1} e(t-\tau)+d e^{\mathrm{T}}(t) R_{2} e(t)-d(1-\dot{d}(t)) e^{\mathrm{T}}(t-d(t)) R_{2} e(t-d(t)) \\
& +\operatorname{Tr}\left\{2 g^{\mathrm{T}}(t, x) P g(t, x)\right\}-2[e(t)-D e(t-\tau)]^{\mathrm{T}} P \Delta L(t) C e(t)+2[e(t)-D e(t-\tau)]^{\mathrm{T}} \\
& \cdot P B_{H}\left[\Delta A(t) x(t)+\Delta A_{d}(t) x(t-d(t))\right] .
\end{aligned}
$$

Then, the following will be obtained by utilizing some inequality techniques

$$
\begin{gathered}
2 x^{\mathrm{T}}(t) P B_{H}\left[\Delta A(t) x(t)+\Delta A_{d}(t) x(t-d(t))\right]=2 x^{\mathrm{T}}(t) P B_{H} E J(t)\left[F x(t)+F_{d} x(t-d(t))\right] \\
\leq \varepsilon_{1}^{-1} x^{\mathrm{T}}(t) P B_{H} E E^{\mathrm{T}} B_{H}^{\mathrm{T}} P x(t)+\varepsilon_{1}\left[F x(t)+F_{d} x(t-d(t))\right]^{\mathrm{T}}\left[F x(t)+F_{d} x(t-d(t))\right], \\
-2 x^{\mathrm{T}}(t-\tau) D^{\mathrm{T}} P B_{H}\left[\Delta A(t) x(t)+\Delta A_{d}(t) x(t-d(t))\right] \leq \varepsilon_{2}^{-1} x^{\mathrm{T}}(t-\tau) D^{\mathrm{T}} P B_{H} E E^{\mathrm{T}} B_{H}^{\mathrm{T}} P D x(t-\tau) \\
+\varepsilon_{2}\left[F x(t)+F_{d} x(t-d(t))\right]^{\mathrm{T}}\left[F x(t)+F_{d} x(t-d(t))\right], \\
2 e^{\mathrm{T}}(t) P B_{H}\left[\Delta A(t) x(t)+\Delta A_{d}(t) x(t-d(t))\right]=e^{\mathrm{T}}(t) P B_{H} E J(t)\left[F x(t)+F_{d} x(t-d(t))\right] \\
\leq \varepsilon_{3}^{-1} e^{\mathrm{T}}(t) P B_{H} E E^{\mathrm{T}} B_{H}^{\mathrm{T}} P e(t)+\varepsilon_{3}\left[F x(t)+F_{d} x(t-d(t))\right]^{\mathrm{T}}\left[F x(t)+F_{d} x(t-d(t))\right],
\end{gathered}
$$

and

$$
\begin{gathered}
-2 e^{\mathrm{T}}(t-\tau) D^{\mathrm{T}} P B_{H}\left[\Delta A(t) x(t)+\Delta A_{d}(t) x(t-d(t))\right] \leq \varepsilon_{4}^{-1} e^{\mathrm{T}}(t-\tau) D^{\mathrm{T}} P B_{H} E E^{\mathrm{T}} B_{H}^{\mathrm{T}} P D e(t-\tau) \\
+\varepsilon_{4}\left[F x(t)+F_{d} x(t-d(t))\right]^{\mathrm{T}}\left[F x(t)+F_{d} x(t-d(t))\right]
\end{gathered}
$$

In addition, it follows that

$$
\begin{gathered}
-2[e(t)-D e(t-\tau)]^{\mathrm{T}} P \Delta L(t) C e(t) \leq \varepsilon_{5}^{-1} e^{\mathrm{T}}(t) P P e(t)+\varepsilon_{6}^{-1} e^{\mathrm{T}}(t-\tau) D^{\mathrm{T}} P P D e(t-\tau) \\
+\left(\varepsilon_{5}+\varepsilon_{6}\right) \delta^{2} e^{\mathrm{T}}(t) C^{\mathrm{T}} C e(t) .
\end{gathered}
$$

With the condition (13), Assumption 3 and Lemma 2.1, one can get

$$
\operatorname{Tr}\left\{g^{\mathrm{T}}(t, x) P g(t, x)\right\} \leq \lambda_{\max }(P)\|M y(t)\|^{2} \leq \varrho x^{\mathrm{T}}(t) C^{\mathrm{T}} M^{\mathrm{T}} M C x(t) .
$$


By incorporating (17)-(22) into (16), it results in

$$
y_{e}^{\mathrm{T}}(t) y_{e}(t)-\gamma^{2} v^{\mathrm{T}}(t) v(t)+\mathcal{L} V(t) \leq \zeta^{\mathrm{T}}(t) \Xi \zeta(t)
$$

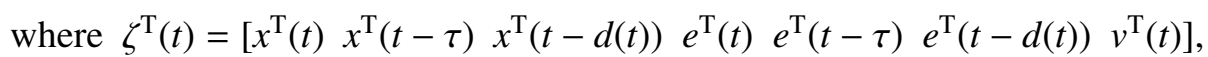

$\Xi=\left[\begin{array}{ccccccc}\Xi_{11} & \Xi_{12} & \Xi_{13} & \Xi_{14} & -(P B K)^{\mathrm{T}} D & 0 & P G_{B} \\ * & \Xi_{22} & -D^{\mathrm{T}} P B_{H} A_{d} & \Xi_{24} & 0 & 0 & -D^{\mathrm{T}} P G_{B} \\ * & * & \Xi_{33} & -\left(P B_{d}\right)^{\mathrm{T}} & \left(P B_{d}\right)^{\mathrm{T}} D & 0 & 0 \\ * & * & * & \Xi_{44} & \Xi_{45} & P A_{d} & P G_{B} \\ * & * & * & * & \Xi_{55} & -D^{\mathrm{T}} P A_{d} & -D^{\mathrm{T}} P G_{B} \\ * & * & * & * & * & \Xi_{66} & 0 \\ * & * & * & * & * & * & -\gamma^{2} I\end{array}\right]$ with

$\Xi_{11}=\operatorname{sys}\{P A+P B K\}+\tau Q_{1}+d Q_{2}+2 \varrho C^{\mathrm{T}} M^{\mathrm{T}} M C+\varepsilon_{1}^{-1} P B_{H} E E^{\mathrm{T}} B_{H}^{\mathrm{T}} P+\left(\varepsilon_{1}+\varepsilon_{2}+\varepsilon_{3}+\varepsilon_{4}\right) F^{\mathrm{T}} F, \Xi_{12}=$ $-[P(A+B K)]^{\mathrm{T}} D, \Xi_{13}=P B_{H} A_{d}+\left(\varepsilon_{1}+\varepsilon_{2}+\varepsilon_{3}+\varepsilon_{4}\right) F^{\mathrm{T}} F_{d}, \Xi_{14}=-P\left(B K+B_{A}\right)+(P B K)^{\mathrm{T}}, \Xi_{22}=-\tau Q_{1}+$ $\varepsilon_{2}^{-1} D^{\mathrm{T}} P B_{H} E E^{\mathrm{T}} B_{H}^{\mathrm{T}} P D, \Xi_{24}=D^{\mathrm{T}} P\left(B K+B_{A}\right), \Xi_{33}=-d(1-\mu) Q_{2}+\left(\varepsilon_{1}+\varepsilon_{2}+\varepsilon_{3}+\varepsilon_{4}\right) F_{d}^{\mathrm{T}} F_{d}, \Xi_{44}=\operatorname{sys}\left\{P\left(A_{B}-\right.\right.$ $L C-B K)\}+\tau R_{1}+d R_{2}+C^{\mathrm{T}} C+\varepsilon_{3}^{-1} P B_{H} E E^{\mathrm{T}} B_{H}^{\mathrm{T}} P+\varepsilon_{5}^{-1} P P+\left(\varepsilon_{5}+\varepsilon_{6}\right) \delta^{2} C^{\mathrm{T}} C, \Xi_{45}=-\left[P\left(A_{B}-L C-B K\right)\right]^{\mathrm{T}} D$, $\Xi_{55}=-\tau R_{1}+\varepsilon_{4}^{-1} D^{\mathrm{T}} P B_{H} E E^{\mathrm{T}} B_{H}^{\mathrm{T}} P D+\varepsilon_{6}^{-1} D^{\mathrm{T}} P P D, \Xi_{66}=-d(1-\mu) R_{2}$.

Then, denote $X=P B K, Y=P L$. By applying the Schur complement, it is shown that $\Xi<0$ is equivalent to the condition (14). In other word, if the LMIs (13) and (14) are established, one can get

$$
\mathcal{L} V(t) \leq-y_{e}^{\mathrm{T}}(t) y_{e}(t)+\gamma^{2} v^{\mathrm{T}}(t) v(t)
$$

Therefore, by taking the zero initial conditions into account, it follows $V(0)=0$. The following inequality can be obtained by integrating (24) with respect to time $t$ from 0 to $\infty$ and taking mathematical expectation of both sides of it, simultaneously, which turns out

$$
0 \leq \mathscr{E}\{V(\infty)\}=\mathscr{E}\left\{\int_{0}^{\infty} \mathcal{L} V(t) \mathrm{d} t\right\} \leq-\mathscr{E}\left\{\int_{0}^{\infty} y_{e}^{\mathrm{T}}(t) y_{e}(t) \mathrm{d} t\right\}+\gamma^{2} \int_{0}^{\infty} v^{\mathrm{T}}(t) v(t) \mathrm{d} t
$$

The inequality indicates $\mathscr{E}\left\{\sup _{0 \neq v(t) \in \mathscr{L}_{2}[0, \infty)}\left\|y_{e}(t)\right\|_{2} /\|v(t)\|_{2}\right\}<\gamma$, i.e., $\mathbf{P 1}$ can be guaranteed.

Next, the stability of the SMDs will be considered with $v(t)=0$. In the position, the following inequality can be easily obtained: $e^{\mathrm{T}}(t) C^{\mathrm{T}} C e(t)+\mathcal{L} V(t)=\xi^{\mathrm{T}}(t) \Theta \xi(t)$,

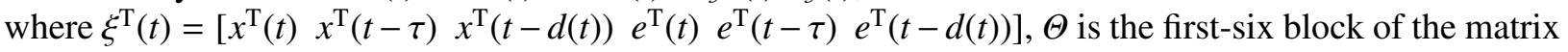
$\Xi$, i.e., it is constituted by the first six lines and six columns of $\Xi$. Obviously, the condition (14) implies that $\Theta<0$, which shows that $\mathscr{L} V(t) \leq \xi^{\mathrm{T}}(t) \Theta \xi(t)<0$, if $\xi(t) \neq 0$. Let $\kappa=\lambda_{\min }\{-\Theta\}>0$, it is followed that

$$
\mathscr{E}\left\{\frac{\mathrm{d} V(t)}{\mathrm{d} t}\right\}=\mathscr{E}\{\mathcal{L} V(t)\} \leq-\kappa \mathscr{E}\left\{\|x(t)\|^{2}\right\}
$$

Moreover, by virtue of $V(t)$, there surely exist finite positive scalars $\alpha_{1}, \alpha_{2}$ such that

$$
\alpha_{1}\|x(t)\|^{2} \leq V(t) \leq \alpha_{2}\|x(t)\|^{2}
$$

which implies $\mathscr{E}\left\{\|x(t)\|^{2}\right\} \geq \alpha_{2}^{-1} \mathscr{E}\{V(t)\}$. Thus, it follows that

$$
\mathscr{E}\left\{\frac{\mathrm{d} V(t)}{\mathrm{d} t}\right\} \leq-\kappa \mathscr{E}\left\{\|x(t)\|^{2}\right\} \leq-\kappa \alpha_{2}^{-1} \mathscr{E}\{V(t)\} .
$$

Further, it is also held that a scalar $\alpha>0$ can be found such that the following can be satisfied:

$$
\mathscr{E}\{V(0)\} \leq \alpha \sup _{-h \leq \theta \leq 0} \mathscr{E}\left\{\|\phi(\theta)\|^{2}\right\} .
$$


By integrating both sides of (27) over the interval $[0, t]$ and invoking (28) as well, it follows that

$$
\mathscr{E}\left\{\|x(t)\|^{2}\right\} \leq \alpha_{1}^{-1} \mathscr{E}\{V(t)\} \leq \alpha_{1}^{-1} \mathscr{E}\{V(0)\} e^{-\kappa \alpha_{2}^{-1} t} \leq \alpha \alpha_{1}^{-1} e^{-\kappa \alpha_{2}^{-1} t} \sup _{-h \leq \theta \leq 0} \mathscr{E}\left\{\|\phi(\theta)\|^{2}\right\}
$$

Denote $\eta=\alpha \alpha_{1}^{-1}$ and $\beta=\kappa \alpha_{2}^{-1}$. The system (10) is exponentially mean-square stable by Definition 1 . In like manner, one can prove the mean-square exponential stability of the system (11), thereby completing the proof.

Remark 6: Theorem 3.1 gives a new criteria for the mean-square exponential stability of the closed-loop systems by employing the SMO approach, which can be regarded as an alternative to the gain matrices $K$ and $L$. It is worth noting that the LMIs (13)-(14) are linear in the set of matrices of $P, Q_{1}, Q_{2}, R_{1}, R_{2}$, $X$ and $Y$, positive scalars $\varrho, \varepsilon_{i}(\mathrm{i}=1,, 6)$ and $\gamma^{2}$, which implies that the scalar $\lambda=\gamma^{2}$ can be included as one of the optimization variables in LMIs (13)-(14) so as to minimize the parameter to reduce the effects of disturbances (i.e., to get the minimum disturbance attenuation level). Then, the optimal solution to the SMO scheme can be obtained by solving the following convex optimization problem:

$$
\min \lambda \text {, subject to }(13)-(14) \text {. }
$$

Remark 7: It should be mentioned that, SMO design for a class of NSS with Markovion jumping parameters has been developed in Kao et al. (2015); and $\mathscr{H}_{\infty}$ integral SMC problem for uncertain neutral systems has been studied based on the state observer in Liu et al. (2015). Yet, the reports did not involve the case that the nonlinearity and/or perturbation appear through the control channel. In this study, the uncertainties or unknown perturbations (i.e., $f(t, x)$ through the control channel of the systems are concerned, and the complicated case can still be handled, whereas Kao et al. (2015); Liu et al. (2015) may not act, which implies the superiority and practicability of the proposed approach.

Remark 8: Now, the general algorithm to solve the LMIs in (13)-(14) which is subjected to the equality constraint (7) will be proposed. Similar to that of Li et al. (2016); Wu et al. (2008), the following optimal minimum problem is summarized to solve the undetermined parameters in Theorem 3.1 as well as the matrices $N_{1}$ and $N_{2}$ in (7):

$$
\begin{array}{r}
\min \eta_{i}(i=1,2), \quad \text { subject to, }(13)-(14), \\
{\left[\begin{array}{cc}
-\eta_{1} I & \left(B^{\mathrm{T}} Z-N_{1} C\right)^{\mathrm{T}} \\
* & -I
\end{array}\right]<0,} \\
{\left[\begin{array}{cc}
-\eta_{2} I & \left(B^{\mathrm{T}} Z D-N_{2} C\right)^{\mathrm{T}} \\
* & -I
\end{array}\right]<0 .}
\end{array}
$$

It is seen that the design is now changed to a minimization problem involving LMIs constraints and linear objective which can be achieved by using the Matlab software. If $\eta_{i}$ equals or tends to zero, the condition (7) can be guaranteed. The verbatim argument is omitted here for brevity.

\subsection{Reachability analysis of sliding mode}

In the following, the attention is focused on an adaptive controller synthesis, by which the sliding mode reachability of the systems trajectories can be ensured so that the system can start its sliding motion to be mean-square exponentially stable with a specific $\mathscr{H}_{\infty}$ disturbance attenuation level. To facilitate the result, the following assumptions are given.

Assumption 4. (Wu et al., 2008) Unknown positive scalar $q$ can be found to satisfy the following inequality:

$$
\|x(t-d(t))\| \leq q\|x(t)\|
$$


Since the system states $x(t)$ are not completely available, the error $e(t)$ may not be computable based on the fact, which is often the case in practical systems. Then, with the relationships among the system states $x(t)$, the error $e(t)$, the outputs $y(t)$ and $\hat{y}(t)$, the following can be valid to a certain degree:

$$
x(t)=C^{+} y(t), \text { and } e(t)=C^{+}(y(t)-\hat{y}(t)) .
$$

Then, the assumption as follows will be reasonably given by togethering with the Assumption 2, 4 and (31).

Assumption 5. Unknown scalars $c_{i}>0(i=1,2)$ can be found to satisfy the following estimation:

$$
\begin{aligned}
\mathscr{Z}= & \left\|H A \left|\||| e(t)\|+\left\|H A_{d}\right\|\|x(t-d(t))\|+\|H \Delta A(t)\|\|x(t)\|\right.\right. \\
& +\left\|H \Delta A_{d}(t)\right\|\|x(t-d(t))\|+\|H B|\|\mid\| f(t, x) \| \\
\leq & c_{1}\|y(t)\|+c_{2}\|\hat{y}(t)\|, t \geq 0 .
\end{aligned}
$$

Notice that the estimation bounds $c_{1}$ and $c_{2}$ are not accessible in design process. Let $\hat{c}_{i}(t)(i=1,2)$ be the associated estimations of them with error parameters being $\tilde{c}_{i}(t)=\hat{c}_{i}(t)-c_{i}$, respectively.

Theorem 3.2: Suppose that the integral sliding surface function is designed by (6), the gain matrix $K$ is obtained by Theorem 3.1. The finite-time reachability of the desirable sliding mode can be guaranteed, if the adaptive SMC law in (33) is synthesized

$$
\begin{aligned}
u(t)= & K \hat{x}(t)-(H B)^{-1}\left[\hat{c}_{1}(t)\|y(t)\|+\hat{c}_{2}(t)\|\hat{y}(t)\|+\|H G\|\|v(t)\|+\rho\right. \\
& \left.+\lambda_{\max }\left(H^{\mathrm{T}} H\right)\|M y(t)\|^{2} /\|s(t)\|\right] \operatorname{sgn}(s(t)),
\end{aligned}
$$

where the updating laws are designed by $\dot{\hat{c}}_{1}(t)=\lambda_{1}\|y(t)\|, \dot{\hat{c}}_{2}(t)=\lambda_{2}\|\hat{y}(t)\|$, and $\lambda_{i}>0(i=1,2)$ are constants as the adaptive gains chosen by the designer, and $\rho$ is a small positive constant. Moreover, the adaptive parameters can be denoted by the integral forms as:

$$
\hat{c}_{1}(t)=\hat{c}_{1}(0)+\lambda_{1} \int_{0}^{t}\|y(\tau)\| \mathrm{d} \tau, \hat{c}_{2}(t)=\hat{c}_{2}(0)+\lambda_{2} \int_{0}^{t}\|\hat{y}(\tau)\| \mathrm{d} \tau
$$

where $\hat{c}_{i}(0)(i=1,2)$ are the initial values of the adaptive estimates, without loss of generality, $\hat{c}_{i}(0)$ can be zero for simplicity.

Proof. Choose a Lyapunov function candidate

$$
\tilde{V}(t, s(t))=\left(s^{\mathrm{T}}(t) s(t)\right)^{\frac{1}{2}}+0.5\left[\lambda_{1}^{-1} \tilde{c}_{1}^{2}(t)+\lambda_{2}^{-1} \tilde{c}_{2}^{2}(t)\right]=\|s(t)\|+0.5\left[\lambda_{1}^{-1} \tilde{c}_{1}^{2}(t)+\lambda_{2}^{-1} \tilde{c}_{2}^{2}(t)\right] .
$$

By the Itô formula, one has

$$
\mathrm{d} \tilde{V}(t, s(t))=\mathcal{L} \tilde{V}(t, s(t)) \mathrm{d} t+\frac{s^{\mathrm{T}}(t)}{\|s(t)\|} H g(t, x(t)) \mathrm{d} \omega(t)
$$

where

$$
\begin{aligned}
\mathcal{L} \tilde{V}(t, s(t))= & \frac{s^{\mathrm{T}}(t)}{\|s(t)\|}\left\{H A e(t)+H A_{d} x(t-d(t))+H \Delta A x(t)+H \Delta A_{d}(t)\right. \\
& \cdot x(t-d(t))+H B[u(t)+f(t, x)]+H G v(t)-H B K \hat{x}(t)\} \\
& \left.+\frac{1}{2} g^{\mathrm{T}}(t, x(t)) H^{\mathrm{T}}\left\{\frac{I_{m}}{\|s(t)\|}-\frac{s(t) s^{\mathrm{T}}(t)}{\|s(t)\|^{3}}\right\} H g(t, x(t))\right) \\
& +\lambda_{1}^{-1} \tilde{c}_{1}(t) \dot{\tilde{c}}_{1}(t)+\lambda_{2}^{-1} \tilde{c}_{2}(t) \dot{\tilde{c}}_{2}(t) .
\end{aligned}
$$


Substituting (33) into (34) and employing some inequality technique yields

$$
\begin{aligned}
\mathcal{L} \tilde{V}(t, s(t))= & \frac{1}{\|s(t)\|} s^{\mathrm{T}}(t)\left\{H A e(t)+H A_{d} x(t-d(t))+H \Delta A x(t)+H \Delta A_{d}(t) x(t-d(t))\right. \\
& +H B f(t, x)+H G v(t)-\left[\hat{c}_{1}(t)\|y(t)\|+\hat{c}_{2}(t)\|\hat{y}(t)\|+\|H G\|\|v(t)\|+\rho\right. \\
& \left.\left.+\lambda_{\max }\left(H^{\mathrm{T}} H\right)\|M y(t)\|^{2} /\|s(t)\|\right] \operatorname{sgn}(s(t))\right\}+\frac{1}{2} g^{\mathrm{T}}(t, x(t)) H^{\mathrm{T}} \\
& \cdot\left\{\frac{I_{m}}{\|s(t)\|}-\frac{s(t) s^{\mathrm{T}}(t)}{\|s(t)\|^{3}}\right\} H g(t, x(t))+\lambda_{1}^{-1} \tilde{c}_{1}(t) \dot{\tilde{c}}_{1}(t)+\lambda_{2}^{-1} \tilde{c}_{2}(t) \dot{\tilde{c}}_{2}(t) \\
\leq & \frac{1}{\|s(t)\|}\|s(t)\|\left\{\|H A\|\|e(t)\|+\left\|H A_{d}\right\|\|x(t-d(t))\|+\|H \Delta A(t)\|\|x(t)\|+\left\|H \Delta A_{d}(t)\right\|\right. \\
& \cdot\|x(t-d(t))\|+\|H B\|\|f(t, x)\|+\|H G v(t)\|\}-\frac{1}{\|s(t)\|}\left[\hat{c}_{1}(t)\|y(t)\|+\hat{c}_{2}(t)\|\hat{y}(t)\|\right. \\
& \left.+\|H G\|\|v(t)\|+\rho+\lambda_{\max }\left(H^{\mathrm{T}} H\right)\|M y(t)\|^{2} /\|s(t)\|\right] s^{\mathrm{T}}(t) \operatorname{sgn}(s(t))+\frac{1}{\|s(t)\|} \\
& \cdot\left\|g^{\mathrm{T}}(t, x(t)) H^{\mathrm{T}} H g(t, x(t))\right\|+\lambda_{1}^{-1} \tilde{c}_{1}(t) \dot{\tilde{c}}_{1}(t)+\lambda_{2}^{-1} \tilde{c}_{2}(t) \dot{\tilde{c}}_{2}(t) \\
\leq & c_{1}\|y(t)\|+c_{2}\|\hat{y}(t)\|+\|H G v(t)\|-\left\{\hat{c}_{1}(t)\|y(t)\|+\hat{c}_{2}(t)\|\hat{y}(t)\|+\|H G\|\|v(t)\|\right. \\
& \left.+\rho+\lambda_{\max }\left(H^{\mathrm{T}} H\right)\|M y(t)\|^{2} /\|s(t)\|\right\}+\frac{1}{\|s(t)\| g^{\mathrm{T}}(t, x(t)) H^{\mathrm{T}} H g(t, x(t)) \|} \\
& +\lambda_{1}^{-1} \tilde{c}_{1}(t) \dot{\tilde{c}}_{1}(t)+\lambda_{2}^{-1} \tilde{c}_{2}(t) \dot{\tilde{c}}_{2}(t) .
\end{aligned}
$$

Notice that the following terms keep valid

$$
\dot{\hat{c}}_{1}(t)=\dot{\tilde{c}}_{1}(t), \quad \dot{\hat{c}}_{2}(t)=\dot{\tilde{c}}_{2}(t) .
$$

Then, in view of Lemma 2.1, and taking (35) into consideration, it follows

$$
\begin{aligned}
\mathcal{L} \tilde{V}(t, s(t)) \leq & c_{1}\|y(t)\|+c_{2}\|\hat{y}(t)\|-\hat{c}_{1}\|y(t)\|-\hat{c}_{2}\|\hat{y}(t)\|-\rho-\lambda_{\max }\left(H^{\mathrm{T}} H\right)\|M y(t)\|^{2} /\|s(t)\| \\
& +\frac{1}{\|s(t)\|}\left\|g^{\mathrm{T}}(t, x(t)) H^{\mathrm{T}} H g(t, x(t))\right\|+\lambda_{1}^{-1} \tilde{c}_{1}(t) \dot{\tilde{c}}_{1}(t)+\lambda_{2}^{-1} \tilde{c}_{2}(t) \dot{\tilde{c}}_{2}(t) \\
\leq & -\tilde{c}_{1}(t)\|y(t)\|-\tilde{c}_{2}(t)\|\hat{y}(t)\|-\rho+\lambda_{1}^{-1} \tilde{c}_{1}(t) \dot{\tilde{c}}_{1}(t)+\lambda_{2}^{-1} \tilde{c}_{2}(t) \dot{\tilde{c}}_{2}(t) \\
= & -\rho .
\end{aligned}
$$

Thus, by integrating (36) from 0 to $t$ and taking expectation for both sides, one can testify that

$$
\mathscr{E}\|s(t)\| \leq \mathscr{E} \tilde{V}(t, s(t)) \leq \mathscr{E} \tilde{V}(0, s(0))-\rho t,
$$

which implies $\mathscr{E}\|s(t)\|=0$ for all $t \geq t_{f}=\frac{\mathscr{E} \tilde{V}(0, s(0))}{\rho}$. Furthermore, it follows that $\|s(t)\|=0$ can be established in finite-time almost surely. The proof is completed.

Remark 9: A novel adaptive sliding mode controller is presented for the NSS, which does not rely on the information of the delayed state, while the design in Kao et al. (2015); Li et al. (2009); Yao et al. (2015) is absolutely a memory controller. Besides, because of the state-dependent stochastic noise in the NSS, the techniques for deterministic neutral systems such as Liu et al. (2015); Wu et al. (2008); Yao et al. (2015) will not be directly applicable to the NSS. Thus, the adaptive controller is synthesized in (33), where the outputs of the systems and its observer are involved based on the information among $x(t), e(t), y(t)$ and $\hat{y}(t)$, by which the unknown bounds $c_{1}$ and $c_{2}$ can be exactly tracked, and the expected performance of the system can be maintained. It is also worth pointing out that, although the control term exists in the error system, it does not mean we need to use it to the error system in simulation and practical applications, i.e., 
the control input is regarded as virtual controller in the error system. This is the fourth highlight of the scheme.

Remark 10: As is seen, a particular state observer has been introduced to estimate the system states, where the observer is designed without any switching terms, and the control input and discontinuous output error injection term (or called the controller compensator) are no longer needed. Based on the observer, the SMC approach is utilized to force the trajectories of the original system and the error system to stay on the predesigned sliding surface almost surely despite the existence of perturbations, thus enabling the closedloop systems to reject the uncertainties and disturbances when specified conditions are satisfied.

In conclusion, the developed observer-based SMC design for the NSS can be seen as a supplement of the SMO strategy.

\section{Illustrative examples}

As is mentioned in the introduction, many physical systems in industrial fields such as lossless transmission lines, collision problem in electrodynamics can be modeled by neutral stochastic differential functional equations when stochastic perturbations are taken into account, which is often inevitable in practical engineering. Thus, the stability analysis and numerical treatment of such systems may be of great importance (Mao, 2007), particularly when there is a need for their control. As a practical application, a general description of the shunted transmission line (see the pages 5 and 6 in Hale.et al. (1993)) was presented, which could be modeled by a type of NSS with the following form:

$$
\mathrm{d}[x(t)-D x(t-\tau)]=m(t, x(t), x(t-\tau)) \mathrm{d} t+n(t) \mathrm{d} \omega(t)
$$

where $\tau=2 / \sqrt{U V}, U$ and $V$ are the mutual capacity and inductance of the line, respectively. In particular, it will be the form of (3) by the selection of

$$
\begin{gathered}
m(t, x(t), x(t-\tau))=(A+\Delta A(t)) x(t)+\left(A_{d}+\Delta A_{d}(t)\right) x(t-\tau)+B[u(t)+f(t, x(t))]+G v(t), \\
n(t)=g(t, x(t)) .
\end{gathered}
$$

Thus, the following Example 1 is firstly introduced with the above discussion.

Example 1. (MIMO system) Let us consider the NSS with the parameters given by Example 4 of Chen et al. (2009) as follows:

$$
\begin{gathered}
D=\left[\begin{array}{cc}
0.1 & 0 \\
0 & 0.1
\end{array}\right], A=\left[\begin{array}{cc}
-0.7 & 0.2 \\
0.3 & 0.1
\end{array}\right], A_{d}=\left[\begin{array}{cc}
0.2 & 0 \\
0.1 & -0.1
\end{array}\right], B=\left[\begin{array}{cc}
-0.2 & 1 \\
0 & 0.7
\end{array}\right], \\
E=F=F_{d}=0, C=\left[\begin{array}{cc}
-0.1 & 0 \\
0.2 & 0.1
\end{array}\right], G=\left[\begin{array}{l}
1 \\
1
\end{array}\right] .
\end{gathered}
$$

The time-delays are chosen by $\tau=d=1$ with $\mu=0$. The additive gain variation of the observer is assumed as $\Delta L(t)=\operatorname{diag}\{0.15 \sin t,-0.2 \sin t\}$, and $\delta$ can be set as 0.2 . With the above parameters, the following solutions are obtained by solving the minimization problem in Remark 8 for $\gamma=0.0517, M=1.25$, and $Z=\left[\begin{array}{cc}2.5 & 1 \\ 1 & 5\end{array}\right]$ as follows:

$\eta_{1}=\eta_{2}=8.0167 \cdot 10^{-16}$ (So, the condition (7) can be ensured), and $N_{1}=\left[\begin{array}{cc}1.0 & -2.0 \\ 58 & 45\end{array}\right], N_{1}=\left[\begin{array}{cc}0.1 & -0.2 \\ 5.8 & 4.5\end{array}\right]$. 


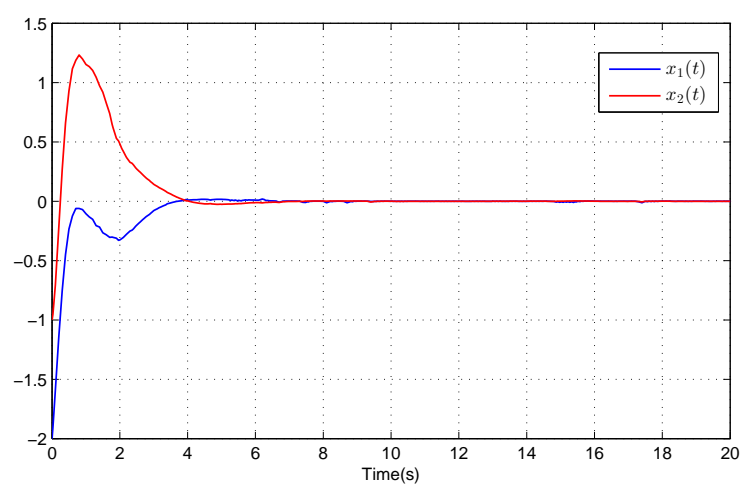

Figure 1. Evolution of the system state

Then, the associated gain matrices can be computed by

$$
L=\left[\begin{array}{cc}
-10.6029 & 14.6571 \\
30.6019 & 34.8072
\end{array}\right], K=\left[\begin{array}{cc}
-6.8782 & -8.4728 \\
-3.0624 & -2.6059
\end{array}\right] \text {. }
$$

At this point, we set the adaptive gains $\lambda_{1}=2.0, \lambda_{2}=2.5$, and $\rho=2.5$. Thus, the integral sliding surface and adaptive sliding mode controller are designed as

$$
\begin{aligned}
s(t)= & N_{1}\left[(y(t)-\hat{y}(t)]-N_{2}[y(t-1)-\hat{y}(t-1)]+\left[\begin{array}{cc}
-0.5 & -0.2 \\
3.2 & 4.5
\end{array}\right]\left[\hat{x}(t)-\left[\begin{array}{cc}
0.1 & 0 \\
0 & 0.1
\end{array}\right] \hat{x}(t-1)\right]\right. \\
& -\int_{0}^{t}\left[\begin{array}{cc}
1.5621 & 0.7005 \\
-15.9343 & -10.0349
\end{array}\right] \hat{x}(\theta) \mathrm{d} \theta
\end{aligned}
$$

and

$$
\begin{aligned}
u(t)= & {\left[\begin{array}{ll}
-6.8782 & -8.4728 \\
-3.0624 & -2.6059
\end{array}\right] \hat{x}(t)+\left[\hat{c}_{1}(t)\|y(t)\|+\hat{c}_{2}(t)\|\hat{y}(t)\|+7.7318\|v(t)\|+2.5\right.} \\
& \left.+47.9619\|y(t)\|^{2} /\|s(t)\|\right] \operatorname{sgn}(s(t))
\end{aligned}
$$

with the updating laws given by

$$
\dot{\hat{c}}_{1}(t)=2.0\|y(t)\|, \quad \dot{\hat{c}}_{2}(t)=2.5\|\hat{y}(t)\| .
$$

Moreover, the system is assumed to subject to the nonlinear perturbation, the external disturbance given by

$$
f(t, x)=\left[\begin{array}{cc}
0 & -\sqrt{3} \cos ^{2} t+1 \\
\sin t-1 & 0
\end{array}\right] x(t), v(t)=-0.15 \sin t \cdot e^{-0.5 t}, \text { and }
$$

the state-dependent stochastic effect gain function $g(t, x)$ is also the same one in Chen et al. (2009). Simulation results are provided in Figs. 1-5 under the initial conditions $x(\theta)=\left[\begin{array}{ll}-2.0 & -1\end{array}\right]^{\mathrm{T}}$, and $\hat{x}(\theta)=\left[\begin{array}{rr}1.5 & -2.5\end{array}\right]^{\mathrm{T}}, \theta \in\left[\begin{array}{ll}-1.0,0 & 0\end{array}\right]$. Among them, Figure 1 and Figure 2 show the responses of the system state and its observer. Plots of the sliding surface function and adaptive SMC law are given by Figure 3 and Figure 4, respectively. Figure 5 depicts the estimated parameters. This example demonstrates the validity of the proposed SMO scheme well. Furthermore, a minimum value of $\gamma$ for which the above system is mean-square exponentially stable with disturbance attenuation is $\gamma_{o p t}=1.8035 \cdot 10^{-7}$. 


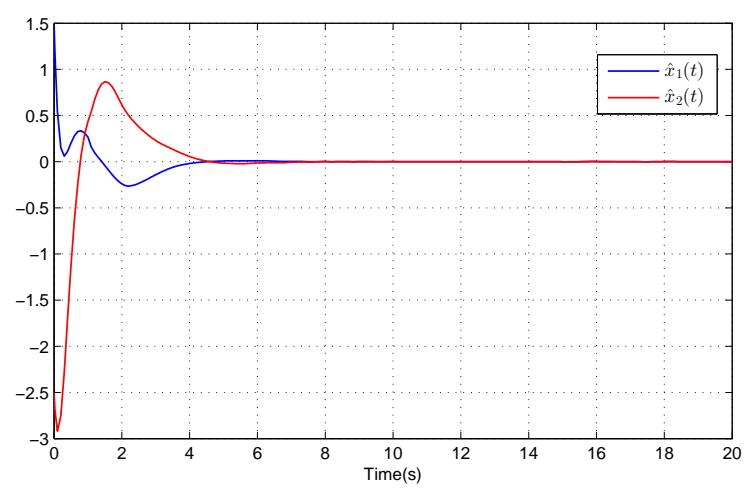

Figure 2. Evolution of the state observer

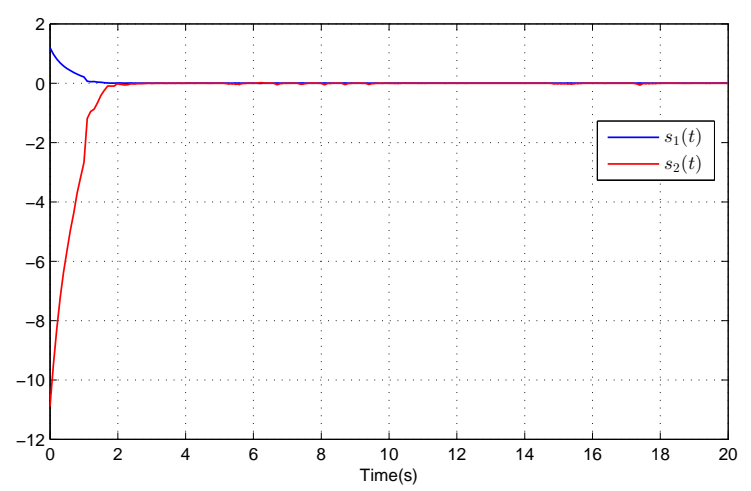

Figure 3. The sliding surface function

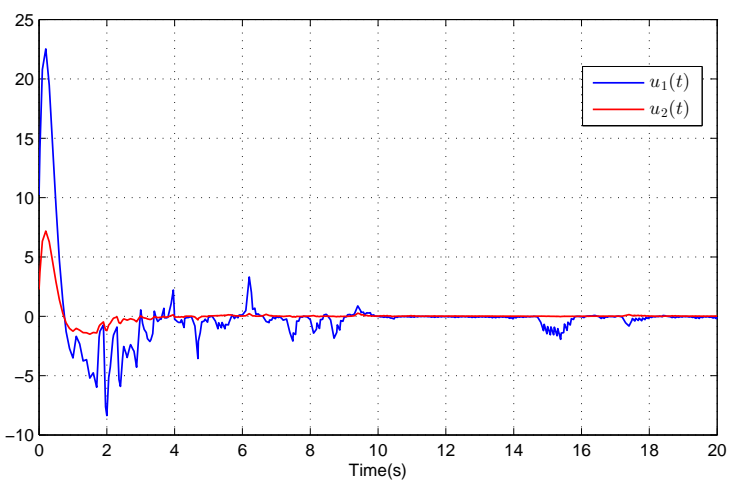

Figure 4. The control inputs

It should be mentioned that, SMC design for uncertain NSS has been studied in Chen et al. (2008), but obviously, it cannot be applied when the states are not measurable, and the complex issue for the SMO design, i.e., uncertainty or perturbation appears through the control channel, is not considered. Also, the robust stochastic $\mathscr{H}_{\infty}$-control problem of uncertain NSS has been studied in Chen et al. (2009), and it could not be utilized for the unavailability of the system states as well. Notice that detailed information of the state-dependent stochastic effect gain function $g(t, x)$ is directly given for their model, whereas it is not needed in our control design, and the desirable performance can be achieved. 


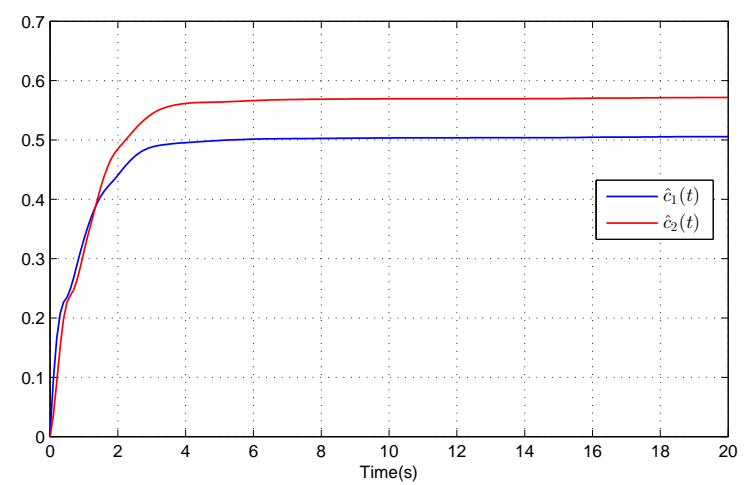

Figure 5. The estimated values

Example 2. (SISO system) The considered uncertain NSS (3) are given with the following data:

$$
\begin{gathered}
D=\left[\begin{array}{cc}
0.1 & 0 \\
0 & 0.1
\end{array}\right], A=\left[\begin{array}{cc}
-2.0 & 2.5 \\
0 & 1.5
\end{array}\right], A_{d}=\left[\begin{array}{cc}
1.0 & 1.5 \\
2.0 & -2.0
\end{array}\right], B=\left[\begin{array}{l}
0 \\
1
\end{array}\right], G=\left[\begin{array}{c}
-0.2 \\
0.1
\end{array}\right], \\
E=\left[\begin{array}{cc}
-0.2 & 0 \\
0 & 0
\end{array}\right], F=\left[\begin{array}{cc}
0.1 & 0 \\
0 & -0.2
\end{array}\right], F_{d}=\left[\begin{array}{cc}
0.2 & 0 \\
0 & 0.1
\end{array}\right], C=\left[\begin{array}{ll}
0 & 1
\end{array}\right] .
\end{gathered}
$$

The time-delays are chosen by $\tau=0.5, d=1$ with $\mu=0$. The additive gain variation of the observer is assumed as $\Delta L(t)=\left[\begin{array}{ll}0.12 \sin t & 0\end{array}\right]^{\mathrm{T}}$, and $\delta$ can be set as 0.12 . For brevity, we select the matrix $Z$ to be the identity matrix, so $N_{1}$ and $N_{2}$ are easily obtained as $N_{1}=1$ and $N_{2}=0.1$. Given $\gamma=0.5212, M=1.5$, the corresponding gain matrices are computed by Theorem 1 as follows

$$
L=\left[\begin{array}{ll}
10.1660 & 62.9185
\end{array}\right]^{\mathrm{T}}, K=\left[\begin{array}{ll}
-7.9271 & -39.9163
\end{array}\right]
$$

Let $\lambda_{1}=2.0, \lambda_{2}=1.5$, and $\rho=1.75$. Hence, the designed sliding surface and adaptive controller are presented by

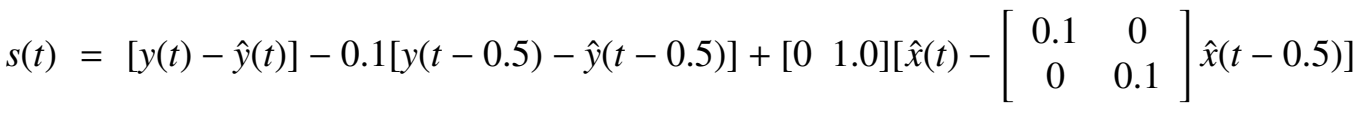

$$
\begin{aligned}
& +\int_{0}^{t}\left[\begin{array}{ll}
7.9271 & 38.4163
\end{array}\right] \hat{x}(\theta) \mathrm{d} \theta .
\end{aligned}
$$

and

$$
\begin{aligned}
u(t)= & {\left[\begin{array}{ll}
-7.9271 & -39.9163]
\end{array} \hat{x}(t)+\left[\hat{c}_{1}(t)\|y(t)\|+\hat{c}_{2}(t)\|\hat{y}(t)\|+0.1\|v(t)\|+1.75\right.\right.} \\
& \left.+1.25\|y(t)\|^{2} /\|s(t)\|\right] \operatorname{sgn}(s(t))
\end{aligned}
$$

with the updating laws given by

$$
\dot{\hat{c}}_{1}(t)=2.0\|y(t)\|, \quad \dot{\hat{c}}_{2}(t)=1.5\|\hat{y}(t)\| .
$$




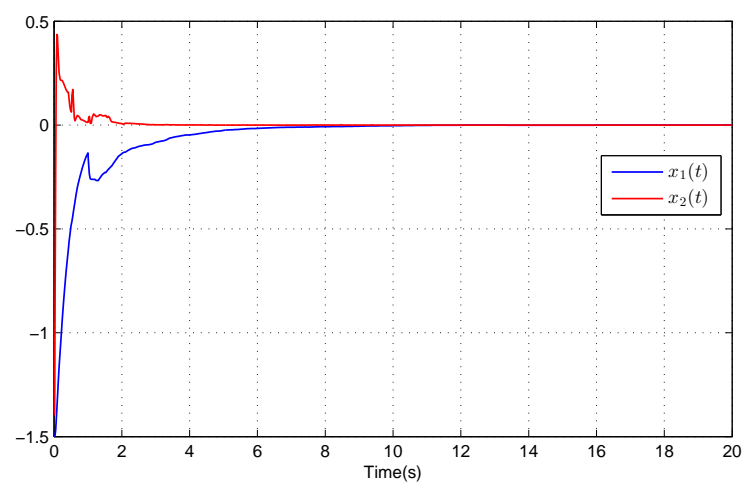

Figure 6. Evolution of the system state

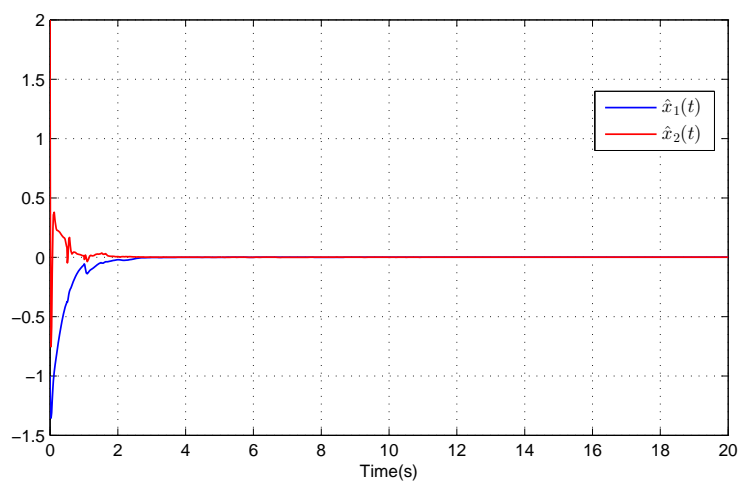

Figure 7. Evolution of the state observer

Given the initial conditions $x(\theta)=\left[\begin{array}{ll}-1.5 & -1.0\end{array}\right]^{\mathrm{T}}$, and $\hat{x}(\theta)=\left[\begin{array}{ll}-1.0 & 2.0\end{array}\right]^{\mathrm{T}}, \theta \in\left[\begin{array}{ll}-1, & 0\end{array}\right]$, and suppose that the system will be exposed by factors, i.e., the nonlinear perturbation, external disturbance and statedependent stochastic effect gain function as below:

$$
f(t, x)=\left[\begin{array}{lll}
-\sqrt{2} \sin ^{2} t & \sqrt{3} \cos t+1
\end{array}\right] x(t), v(t)=\sin t /\left(t^{2}+1.5\right), \text { and } g(t, x)=\left[\begin{array}{ll}
0.25 & -0.5
\end{array}\right] x(t),
$$

respectively. The simulations of the closed-loop system (3)-(5) are shown in Figs. 6-10, which show our design goals have been satisfied.

It should be pointed out that, the observer-based SMC design in Li et al. (2009) cannot be applied to this example obviously, with the fact that the parameters of the state-dependent stochastic effect gain function $g(t, x)$ are not clearly provided in advance. Also, the case that uncertainty or perturbation may exist through the control channel was not investigated in the results of Kao et al. (2015); Li et al. (2009).

Therefore, all of these situations may reflect the effectiveness and superiority of the proposed method well, and the range is extended for dealing with control problem of the NSS.

\section{Conclusions}

The problem of mean-square exponential stability for a class of uncertain NSS with $\mathscr{H}_{\infty}$ disturbance attenuation level has been investigated via a novel SMO approach in the paper. The non-fragile state observer has been simplified to estimate the states of the system, based on which a novel integral-type sliding surface 


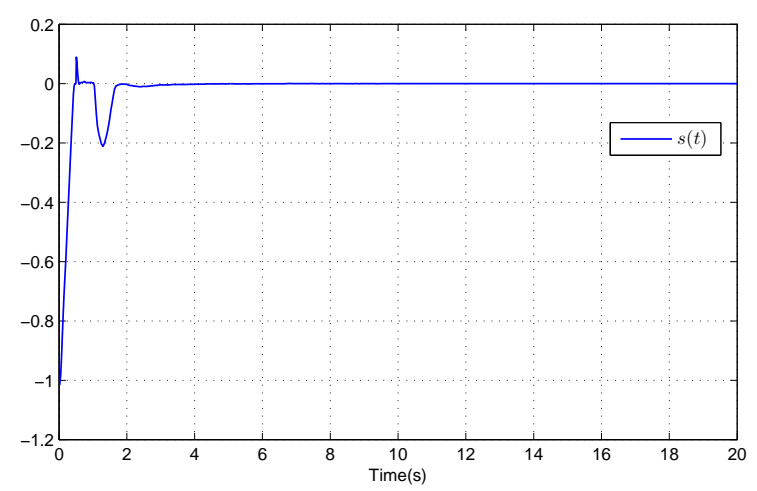

Figure 8. The sliding surface function

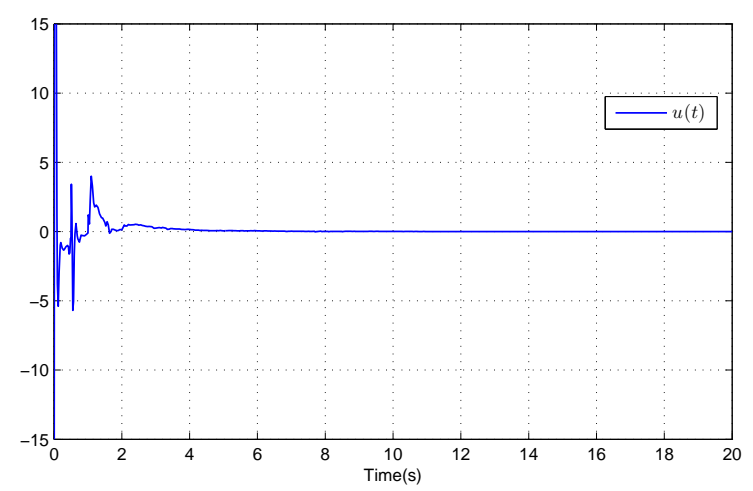

Figure 9. The control inputs

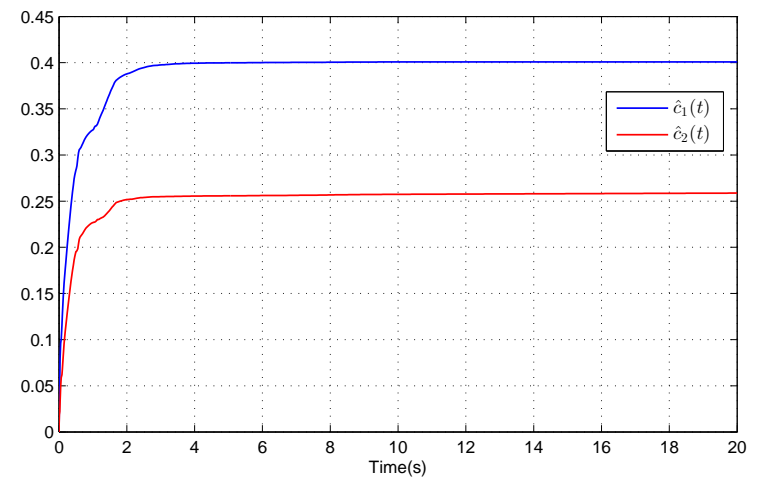

Figure 10. The estimated values

has been established to achieve the entire control scheme. Then, the sufficient condition for the $\mathscr{H}_{\infty}$ performance and mean-square exponential stability of the resultant SMDs of the closed-loop systems has been derived via LMI. By utilizing the novel adaptive SMC law, the finite-time reachability of the predesigned sliding surface has been ensured. Finally, simulation examples have been provided to show the validity and superiority of the proposed scheme. This also provides an alternative method to study the neutral stochastic control systems in future research.

It should be mentioned that, the current study also assumes to be a linear model even though all the 
nonlinearity can be put into uncertainty and tackled via the proposed method. However, if a non-affine model (for example nonlinear rational model or called total nonlinear model, see Zhu, Wang, Zhao, Li, \& Billingse (2015)) or with time-delay is considered, it is still an open and challenging issue for future directions. The problems will be investigated via some novel approaches, e.g., U-block model-based design (Zhu, Zhao, \& Zhang, 2016) in our future research.

\section{Disclosure statement}

No potential conflict of interest was reported by the authors.

\section{Funding}

The authors would like to thank the editors and the anonymous reviewers for their constructive comments which helped to improve the quality and presentation of this paper. This work was partially supported by the National Natural Science Foundation of China [grant number 61273188], [grant number 61374079], [grant number 61473097], [grant number 61603204], [grant number 41306002], the Natural Science Foundation of Shandong province [grant number ZR2016FP03] and the Qingdao Application Basic Research Project [grant number 16-5-1-22-jch].

\section{References}

Ahmad, A., \& Zhu, Q. (2015). Advances and applications in sliding mode control systems. Springer: Berlin.

Basin, M., Ferreira, A, \& Fridman, L. (2007). Sliding mode identification and control for linear uncertain stochastic systems. International Journal of Systems Science, 38(11), 861-869.

Chen, D., \& Zhang, W. (2008) Sliding mode control of uncertain neutral stochastic systems with multiple delays. Mathematical Problems in Engineering, (2008), Article ID 761342, 9 pages.

Chen, H., Hu, P., \& Wang, J. (2014) Delay-dependent exponential stability for neutral stochastic system with multiple time-varying delays. IET Control Theory \& Applications, 8(17): 2092-2101.

Chen, G., \& Shen, Y. (2009) Robust $\mathscr{H}_{\infty}$ filter design for neutral stochastic uncertain systems with time-varying delay. Journal of Mathematical Analysis and Applications, 353(1): 196-204.

Chen, W., Zheng, W., \& Shen, Y. (2009). Delay-dependent stochastic stability and $\mathscr{H}_{\infty}$-control of uncertain neutral stochastic systems with time delay. IEEE Transactions on Automatic Control, 54(7), 1660-1667.

Chen, Y., Zheng, W., \& Xue, A. (2010). A new result on sbility analysis for stochastic neutral systems. Automatica, 46(12), 2100-2104.

Elhsoumi, A., Ali, H., Bel, S., Harabi, R., \& Abdelkrim, M. (2016). Unknown input fault detection and isolation observer design for neutral systems. Asian Journal of Control, doi: 10.1002/asjc. 1256.

Gao, C., Liu, Z., \& Xu, R. (2013). On exponential stabilization for a class of neutral-type systems with parameter uncertainties: An integral sliding mode approach. Applied Mathematics and Computation, 219(23), 11044-11055.

Hale, J., \& Sjoerd M. Verduyn Lunel. (1993). Introduction to Functional Differential Equations. New York: SpringerVerlag, USA.

Huang, L., \& Mao, X. (2009). Delay-dependent exponential stability of neutral stochastic delay systems. IEEE Transactions on Automatic Control, 54(1), 147-152.

Huang, L., \& Mao, X. (2010). SMC design for robust $\mathscr{H}_{\infty}$ control of uncertain stochastic delay systems. Automatica, 46(2), 405-412.

Hung, J., Gao, W., \& Hung, J. (1993). Variable structure control: a survey. IEEE Transactions on Industrial Electronics, 40(1), 2-22.

Jankovic, S., Randjelovic, J., \& Jovanovic, M. (2009). Razumikhin-type exponential stability criteria of neutral stochastic functional differential equations. Journal of Mathematical Analysis and Applications, 355(2), 811-820.

Kao, Y., Li, W., \& Wang, C. (2014). Nonfragile observer-based $\mathscr{H}_{\infty}$ sliding mode control for Itô stochastic systems with Markovian switching. International Journal of Robust and Nonlinear Control, 24(15), 2035-2047.

Kao, Y., Wang, C., Xie, J., Karimi, HR., \& Li, W. (2015). $\mathscr{H}_{\infty}$ sliding mode control for uncertain neutral-type stochastic systems with Markovian jumping parameters. Information Sciences, 314, 200-211.

Kao, Y., Xie, J., Wang, C., \& Karimi, HR. (2015). A sliding mode approach to $\mathscr{H}_{\infty}$ non-fragile observer-based control 
design for uncertain Markovian neutral-type stochastic systems. Automatica, 52, 218-226.

Karimi, HR. (2011). Robust delay-dependent control of uncertain time-delay systems with mixed neutral, discrete, and distributed time-delays and Markovian switching parameters. IEEE Transactions on Circuits and Systems I: Regular Papers, 58(8), 1910-1923.

Li, H., Shi, P., Yao, D., \& Wu, L. (2016). Observer-based adaptive sliding mode control for nonlinear Markovian jump systems. Automatica, 64, 133-142.

Li, Q., \& Li, W. (2009) Robust observer design for uncertain Itô neutral stochastic time-delay systems via sliding mode control. Journal of Mathematical Sciences, 161(2): 283-296.

Lin, C., Wang, Q., Lee, T., He, Y., \& Chen, B. (2008). Observer-based $\mathscr{H}_{\infty}$ fuzzy control design for T-S fuzzy systems with state delays. Automatica, 44(3), 868-874.

Liu, Z., Gao, C., \& Kao, Y. (2015). Robust H-infinity control for a class of neutral-type systems via sliding mode observer. Applied Mathematics and Computation, 271, 669-681.

Liu, Z., \& Gao, C. (2016) A new result on robust $\mathscr{H}_{\infty}$ control for uncertain time-delay singular systems via sliding mode control. Complexity, doi:10.1002/cplx.21793.

Mao, X. (2007). Stochastic differential equations and their applications (2nd ed.). Chichester: Horwood Publishing.

Niu, Y., Lam, J., \& Wang, X. (2004). Sliding-mode control for uncertain neutral delay systems. IEE ProceedingsControl Theory and Applications, 151(1), 38-44.

Parlakc, M. (2010). Robust delay-dependent guaranteed cost controller design for uncertain nonlinear neutral systems with time-varying state delays. International Journal of Robust and Nonlinear Control, 20(3), 334-345.

Qiao, F., Zhang, Y., Zhu, Q., \& Zhang, H. (2009). Adaptive sliding mode observer for non-linear stochastic systems with uncertainties, International Journal of Modelling, Identification and Control, 8(1), 18-24.

Rahme, S., \& Meskin, N. (2015). Adaptive sliding mode observer for sensor fault diagnosis of an industrial gas turbine. Control Engineering Practice, 38, 57-74.

Sakthivel, R., Mathiyalagan, K., \& Anthoni, S. (2012). Robust stability and control for uncertain neutral time delay systems. International Journal of Control, 85(4), 373-383.

Shen, H., Xu, S., Zhou, J., \& Lu, J. (2011). Fuzzy $\mathscr{H}_{\infty}$ filtering for nonlinear Markovian jump neutral systems. International Journal of Systems Science, 42(5), 767-780.

Shi, P., Liu, M., \& Zhang, L. (2015). Fault-tolerant sliding-mode-observer synthesis of Markovian jump systems using quantized measurements. IEEE Transactions on Industrial Electronics, 62(9), 5910-5918.

Song, B., Xu, S., Xia, J., Zou, Y, \& Chen, Q. (2011). Design of robust $\mathscr{H}_{\infty}$ filters for a class of uncertain nonlinear neutral stochastic systems with time delays. International Journal of Systems Science, 42(4), 633-642.

Song, B., Park, J., Wu, Z., \& Zhang, Y. (2013) New results on delay-dependent stability analysis for neutral stochastic delay systems. Journal of the Franklin Institute, 350(4): 840-852.

Utikin, V. (1992). Sliding mode in optimization and control. Springer: Berlin.

Wu, L., \& Lam, J. (2008) Sliding mode control of switched hybrid systems with time-varying delay. International Journal of Adaptive Control and Signal Processing, 22(10): 909-931.

Wu, L., Wang, C., \& Zeng, Q. (2008). Observer-based sliding mode control for a class of uncertain nonlinear neutral delay systems. Journal of the Franklin Institute, 345(3), 233-253.

Xu, S., Chu, Y., Lu, J., \& Zou, Y. (2006) Exponential dynamic output feedback controller design for stochastic neutral systems with distributed delays. IEEE Transactions on Systems, Man, and Cybernetics-Part A: Systems and Humans, 36(3): 540-548.

Xu, S., Shi, P., Chu, Y., \& Zou, Y. (2006) Robust stochastic stabilization and $\mathscr{H}_{\infty}$ control of uncertain neutral stochastic time-delay systems. Journal of Mathematical Analysis and Applications, 314(1): 1-16.

Yan, X., Spurgeon, S., \& Edwards, C. (2010). Sliding mode control for time-varying delayed systems based on a reduced-order observer. Automatica, 46(8), 1354-1362.

Yao, D., Liu, M., Li, H., \& Ma, H. (2015). Robust Adaptive sliding mode control for nonlinear uncertain neutral Markovian jump systems. Circuits Systems and Signal Processing, doi:10.1007/s00034-015-0171-9.

Zhao, D., \& Zhu, Q. (2014). Position synchronised control of multiple robotic manipulators based on integral sliding mode. International Journal of Systems Science, 45(3), 556-570.

Zhang, J., Shi, P., \& Lin, W. (2016). Extended sliding mode observer based control for Markovian jump linear systems with disturbances. Automatica, 70, 140-147.

Zhao, L., \& Jia, Y. (2015). Finite-time attitude tracking control for a rigid spacecraft using time-varying terminal sliding mode techniques. International Journal of Control, 88(6), 1150-1162.

Zhu, Q., Wang, Y., Zhao, D., Li, S., \& Billingse, S. (2015). Review of rational (total) nonlinear dynamic system modelling, identification, and control. International Journal of Systems Science, 46(12), 2122-2133. 
Zhu, Q., Zhao, D., \& Zhang, J. (2016). A general U-block model-based design procedure for nonlinear polynomial control systems. International Journal of Systems Science, 47(14), 3465-3475. and 